\section{Die elektrolytische Darstellung von Legierungen aus wässerigen Lösungen ${ }^{1}$ ).}

\author{
Ton Prof. Di. Roberf Kreman, Gort.
}

Dic hauptsächlichste technische Anwendung indet die Abscheidung einzelner Metalle in der Galvanostegie, wo es sich darum handelt, Gegenstände, vornehmlich solche aus Eisen mit mehx oder minder dünnen, festhaftenden Häutchen anderer Netalle zu überziehen. Teils handelt es sich hierbei darum, Rostschutz zu erzielen, teils der unscheinbaren Grundmasse oberflächlich den hellen Glanz anderer Metalle zu erteilen. Einmal, $1 \mathrm{~m}$ nun bestimmte Farbtöne $\mathrm{zu}$ erzielen, ander. seits auch, um an einem teuren Metall, wie z. B. Silber, Platin zu sparen, sowie auch die mechanisclien Eigenschaften des Uberzuges zu verbessern, hat sich das Bedürfnis herausgestellt, Ưberzüge aus gleichzeitig zwei Metallen herzustellen. Diese Uberzüge aus zwei Metallen werden dann härter sein, als die einzelnen Komponenten, und auch oft eine gröBere Resistenz gegen oxydierende Einflüsse aufweisen, wenn sie nicht in Form eines mechanischen Gemenges abgeschieden werden, sondern in Form von Verbindungen bzw. festen Lösungen der beiden Metalle.

Auf die Möglichkeit der Bildung solcher bei gewöhnlicher Temperatur, bei Abscheidung eines, gegebenenfalls beider Metalle aus dem Ionenzustand haben wir bereits im forigen Jahrgange, Heft $34 / 35$, S. 841, dieser Zeitschrift hingewiesen.

Das Bedürfnis der Technik nach der gleichzeitigen Abscheidung zweier Metalle auf einer" Grundmasse illustrieren die Vorschläge, aus Einzelbädern die einzelnen Metalle nacheinander in Form dünner Schichten niederzuschlagen und das aus abwechselnd dünnen Schichten der beiden Metalle bestehende mechanische Gemenge durch nachheriges Erhitzen auf Rotglut zu legieren.

Es ist nun naheliegend, zur Vereinfachung des Verfahrens beide Metalle gleichzeitig aus einem Bad, das die Salze, also die Ionen der beiden Metalle enthält, abzuscheiden.

Es erhebt sich nun die Frage, unter welchen Bedingungen wir dic Ionen zweier Metalle, die sich in Lösung befinden, gleichzeitig zur Abscheilung bringen können. Genügt es etwa, einfach beliebige Mischungen zweier Salzlösungen zu elektrolysiexen?

Zux Beantwortung dieser Frage seien kurz die GesetzmäBigkeiten bei der Abscheidung eines einzelnen Metalles in Erinnerung gebracht.

Bekanntlich besteht zwischen jedem Metall und der wässerigen Lösung seiner Ionen ein Potentialunterschied, den wir in Volt messen. Der. selbe ist abhängig von der individuellen Lösungstension $P_{m}$ des Metalles und dem entgegengesetzt gerichteten osmotischen Druck $p_{\text {in }}$ der Ionen die-

1) Auszug aus einem Vortrag, gehalten am 5. Juni 1914 in der chemischen Sektion des naturwisken. schaftlichen Vereing fî̀r Steiermark. ses Metalles, den wir der Konzentration der betreffenden Ionen proportional setzen können. Es läBt sich für Zimmertemperatur für diesen Potentialsprung $\mathrm{E}_{\mathrm{m}}$ die Formel:

$$
\mathrm{E}_{\mathrm{m}}=\frac{0,058}{\mathrm{n}} \log \frac{\mathrm{P}_{\mathrm{m}}}{\mathrm{p}_{\mathrm{m}}}
$$

ableiten. Ist $p_{m}=P_{m}$, wird $\mathrm{E}_{m}=0$. Ist $p_{m}>P_{m}$, zählen wir $E_{m}$ positiv und ungekehrt, ist $\mathrm{p}_{\mathrm{m}}<\mathrm{P}_{\mathrm{m}}$, zählen wir $\mathrm{E}_{\mathrm{m}}$ negativ. Als $\mathrm{Null}$ wert des Potentials sprechen wir das Potential an, das eine von Wasserstoff unter dem Druck von 1 Atmosphäre bespälte platinierte Platinelektrode gegen eine Lösung von $1 \mathrm{nH}$-Ionenkonzentration zeigt. Beziehen wir uns auf eine solche Elektrode, erhalten wir für einzelne Metalle gegen die $1 \mathrm{n}$ Lösungen ihrer Ionen folgende Werte:

$$
\begin{array}{cccccr}
\mathrm{Ag} & \mathrm{Cu} & \mathrm{H}_{2} & \mathrm{Fe} & \mathrm{Zn} & \mathrm{Mg} \\
+0,77 & +0,33 & \pm 0,0 & -0,46 & -0,80 & -1,49
\end{array}
$$

Durch die obige Formel wird aber auch in erster Linie der Abscheidungsvorgang eines Metalles an der Kathode geregelt, d. h., um an der Kathode ein bestimmtes Metall zur Abscheidung zu bringen, müssen wir der Kathode zum mindesten diejenige Spannung aufzwingen, die dem Gleichgewichtspotential - Metall - Elektrolyt entspricht. Ist die Spannung kleiner, tritt infolge der auftretenden entgegengesetzt gerichteten Polarisationsspannung kein Stromdurchgang ein. Erst wenn die Polarisationsspannung überwunden ist, tritt Stromdurchgang und damit Abscheidung des betreffenden Metalles an der Kathode ein. Dieser Zersetzungspunkt ist also dem Gleichgewichtspotential identisch, Gleichgewicht vorausgesetzt, und entspricht einem Stromdurchgang von unendlich kleiner Intensität oder, wenn wir die Stromstärke auf die Flächeneinheit bezogen mit Stromdichte bezeichnen, einer Stromdichte von praktisch annähernd Null.

Was geschieht nun, wemn wir durch Erhöhung der angelegten Spannung die Stromdichte steigern? Mit steigender Stromdichte wird nach dem Faradayschen Gesetz in der Zeiteinheit eine immer größere Anzahl von Ionen abgeschieden. Damit geht Hand in Hand eine mit steigender Stromdichte steigende Verarmung an Ionen in der Nähe der betreffenden Elektrode. Das heißt, es wird in der Formel $p_{m}$ kleiner und damit mit steigender Stromdichte der Wert $\mathrm{E}_{\mathrm{m}}$ nach der unedlen Seite verschoben; es sind also mit steigender Stromdichte immer höhere Kathodenpotentiale zur Abscheidung des Metalles nötig. Nun ändert sich aber $\mathrm{E}_{m}$ nur mit dem Logarithmus von $\mathrm{p}_{\mathrm{m}}$; es werden also relativ großen Anderungen von $\mathbf{p}_{\mathrm{m}}$ relativ kleine Änderungen von $\mathrm{E}_{\mathrm{m}}$ entsprechen, falls, was wir zunächst annehmen, die Anderungen von $\mathrm{E}_{\mathrm{m}}$ mit der Stromdichte lediglich durch Konzentrationspolarisation bedingt sind.

Denken wir uns auf Grund dieser tberlegungen die Abhängigkeit der Kathodenpotentiale von der Stromdichte für einige Metalle $A, B, C$ ete. 
In cinem Koordiuatensyoten aufgetragen, so sehen wir, dab die Kurven, ansgehend ron den Werten der Gleichgewichtspotentiale, die ja der Stromdichte Null entsprechen, steil ansteigen.

Denken wir uns nun in einer Lösung die Ionen dieser drei Metalle gleichreitig in Lösung. Wenn wir nun mit unendlich kleinen Stromdichten ar" beiten, so wird zunächst das Kathodenpotential des edelsten Metalles A erreicht und es ritt lediglich Abscheidung an diesem Netall ein. Erst wenn dieses nahezu vollständig ausgefinllt ist, das uächstunedlere usf. Mit steigender Stromdichte änderı sich aber diese Verhältnisse. Von einer Stromdichte 00 an (siehe Fig. 1) wixd das Kathodenpotential erreicht bzw. überschritten, das zur gleichzeitigen Ausscheidung ron $B$ auspeicht, d. h. uber Stromdichten ron 00 an scheiden sich beide Metalle gleichzeitig aus. Sei durch die Stromdichte $z^{\prime}{ }^{\prime}$ die endlich erreichbare Strondichte gegeben, so

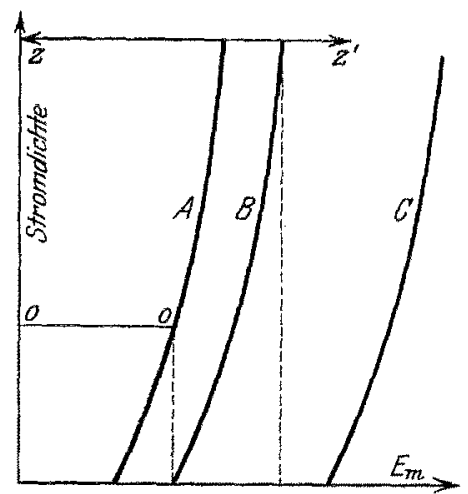

Hig. 1.

sthen wir, daß es unmöglich ist, neben $A$ oder neben $B$ gleichzeitig das Metall $\mathrm{C}$ aus wässeriger Lösung abzuscheiden, weil eben das zur Abscheidung von $\mathrm{C}$ nötige Kathodenpotential bei endlichen Stromdichten nicht erreicht wird.

Wir können also zusammenfassend sagen: Um zwei oder mehrere Metalle abruscheiden, müssen die Stromdichtepotentialkurren genigend nahe bei einander licgen.

Bei. den allermeisten Netalleu liegen aber die Stromdichtepotentialkurven ziemlich weit entfernt von einander und es erhebt sich die Frage, wie wir eine Näherung derselben herbeiführen können.

Kehren wir zu unserer Formel zurück.

Wir können ein Metall gegen die Lösung seiner Ionen unedler machen, wenn wir $\mathrm{p}_{\mathrm{m}}$ kleiner machen. Dies können wir durch Zusätze erzielen, die mit dem betreffenden Metallion komplexe Tonen bilden, also die Konzentration und damit den osmotischen Druck der einfachen Tonen bedeutend herabsetzen und damit $\mathrm{E}_{\mathrm{m}}$ nach der elektronegativen Seite verschieben. In Lösungen von

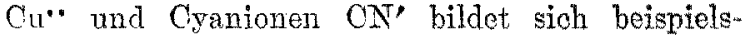

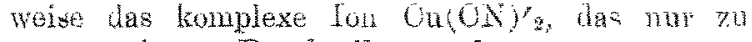
ganz gexingen Bruchteilen nach

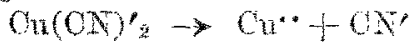

xevfallen ist. Demgemal exschemt in einer Lasung, die z. B. 1 Hol CuCN + 2 Nol KON enthalt, das Potential $\mathrm{Cu} / \mathrm{Cu}^{*}$ gegenuber dem Werte in 1 n Cu* Ionenkonzentration nu mand 0,9 Volt nach der medlen Soito rerschoben: es heträgt $-0,601$ Volt.

Ton diesem Punkte aus rerläuf die Strom. dichtepotentialkurve des Kupfers in einer Lösung obiger Definition. Sie würde also der Stromdichtepotentialkurve der Zinkabseheidung aus Zinksulfat erheblich nähel kommen. Wenn wir aber Lösungen von Kupfer- und Zinksalzeñ mit entsprechendem KON-Übersohub verwenden, erlejdet abs]: auch die Stromdichtepotentialkurve des Zinks eine Verschiebung nach der unedlen Seite, weil auch Zink mit ION Komplexionen hildet. die nach dem Schema:

$$
\mathrm{Zn}(\mathrm{CN})^{\prime \prime}{ }_{4} \rightarrow \mathrm{Zni} \cdot+4 \mathrm{CN}
$$

diswociert sind. Cet. parib, also in einer Lösung, die auf $1 \mathrm{Mol} \mathrm{Zn}(\mathrm{ON})_{2}, 2 \mathrm{Mol} \mathrm{KON}$ enthält, ist de.. Dissociationsgrad der komplexen Ionen, d. h. die Konzentration der freien Metallionen riel gröBer. So beträgt der Unterschied der Gleichgewichtspotentiale dieser Lösung gegen die Zinksulfatlösung nur 0.2 Volt, indem der Potentialsprung $\mathrm{Zn} / \mathrm{Zn}(\mathrm{ON})_{2}+2 \mathrm{KON}=-1,03$ Volt heträgt.

Infolge des geringeren Komplexitätsgrades des unedleren Metalls also wird mit steigendem Zusatr. von KON eine Annäherung der Stromdichtepotenlialkurven zu erzielen sein, die mit steigendem $\mathrm{Zn-}$ satz des Komplexbildners eine immer weitergehende wird. So werden z. B. bei 25 fachem Uberschuß an KON die Gleichgewichtspotentiale von Kupfer und Zink gleich (etwa bei rund -1,3 Volt), und daribler hinaus erscheint das Kupfer sogar unedler als das Zink.

Bei einer solchen Annaherung der Stromdichtepotentialkurven durch Zusatz von Komplexsalzen müssen wir abez bedenken, dab bei diesen Versehiebungen die Stromdichtepotentialkurven in immer elektronegativere Gebiete zu liegen kommen. In neutralen Lösungen, wo die H-Ionenkonzentration ja nur eine sehr geringe ist, liegt das Potential Is/H-Tonen an platinierten Platinelektroden bei erheblich elektronegativen Werten bei $-0,41$ Volt; von diesem Punkt aus steigt also die Stromdichtepotentialkurve der Wasserstoffabscheidung an Platinelektroden etwa in der in Fig. 1 skizzierten Weise an.

Wir kämen also zum SchluB, daß, sobald für die Abscheidung irgend eines Netalles in einer Lösung Kathodenpotentiale nötig sind, die unedler sind, als der Wasserstoffabscheidung entspricht, die Metallabscheidung nicht gelingt.

Nun wissen wir aber, daB dies z. B. für die Magnesium- und Kaliumabscheidung wohl zutrifft, nicht menr aber für die Abscheidung des Fisens, des Zinks. 
Dies hat seinen Grund darin, daß der Wasserstoff nur an platinierten Platinelektroden reversible Potentiale zeigt, d. h. nur an solchen Zersetzungs- und Gleichgewichtspotentialen identisch sind.

An andern Metallen erleidet die Wasserstoffabscheidung jedoch eine Verzögerung, welche die $A b$ scheidung erst an weit höheren Kathodenpotentialen ermöglicht. Den Untersehied zwischen dem Gleichgewichtspotential $\mathrm{H}_{2} / \mathrm{H}$-Ionen und dem zur Abscheidung nötigen Kathodenpotential bezeichnen wir als Uberspannung des Wasserstoffs $\eta_{k} \cdot r_{1}$ ist ron Temperatur und Stromdichte abhängig und variiert von Kathodenmetall zu Metall. Besonders hohe Werte rund von $1 / 2$ Volt zeigt $r_{1}$ an Quecksilberkathoden. Wir müssen also, wenn wir die Formel für das Potential $\mathrm{H}_{2} / \mathrm{H}$-Tonen allgemeingültig machen wollen, $\eta$ als Korrektionsglied einfügen und können sagen: Ist in der Formel:

$$
\mathrm{E}_{\mathrm{H}}=\frac{0,058}{\mathrm{n}} \log \cdot \frac{\mathrm{P}_{\mathrm{H}}}{\mathrm{p}_{\mathrm{H}}}+\eta
$$

bei der betreffenden Stromdichte $\mathrm{E}_{\mathrm{m}}<\mathrm{E}_{\mathrm{H}}$, tritt Metallabscheidung ein; ist das tingekehrte der Fall, so superponiert die Wasserstoffabscheidung. Wir werden also bei der Wahl der Versuchsbedingungen darauf $z u$ achten haben, dal wir nicht Potentiale erreichen, die unedler sind, als der Wasserabscheidung unter gegebenen Umständen entspricht. So tritt z. B. in einer Lösung, in der Gleichheit der Potentiale von Kupfer und Zink eingetreten ist, bei - 1,3 Volt keine Metallabscheidung mehr ein, sondern lediglich Wasserstoffabscheidung.

Nicht unter allen Umständen verlaufen aber die Stromdichtepotentialkurven in der Weise, wie es die lediglich spezifische Beeinflussung dureh Konzentrationspolarisation bedingen wïrde, nämlich direkt steil ansteigend, wie es in Fig. 1 angenommen wurde. Vor allem bei Abscheidungen aus komplexen Salzlösungen kommt es häufig vor, daß mit steigender Stromdichte auch große Änderungen des Kathodenpotentials Hand in Hand gehen; d. h. in solchen Fallen erweisen sich die Kathoden erheblich polarisierbar.

Einen solchen Fall beobachten wir z. B. bei der Stromdichtepotentialkurve von $\mathrm{Ou} /$ in $\mathrm{Cu}(\mathrm{ON})_{2} 2$ KCN-Lösung bei gewöhnlicher Temperatur (Kurve 1 in Fig. 2). Die Erklärung für einen. solchen Verlauf kann man in dem Umstand sehen, Jal die Nachbildung der: für' dex Abscheidungsvorgang in Betracht kommenilun Kupferionen aus deu Komplexionen bei gewöhnlicher Temperatur: so langsam verläuft, daß sich in der Nähe der: Ka= thode nicht momentan das Gleichgewicht ein. stellt und eine noch viel größere Verarmung vou Kupferionen in der Nähe der Kathode sich ein. stellt, als $z$. B. bei der Abscheidung aus Sulfatbädern.

Für diese Errklärung spricht der Vmstand, dals die Stromdichtepotentialkurven dor Silberabschej- dung aus komplexen Silbercyanionen, deren Zerfallsgeschwindigkeit nachweislich viel größer als die des lkomplexen Kupfercyanions ist, auch bei gewöhnlicher Temperatur normal verläuft, sowie auch die Tatsache, daB abnorme Kurven eine $A n-$ näherung an den normalen Verlauf bei höherer Temperatur erfahren, wo die Reaktionsgeschwin" digkeit des metallionennachbildenden $\nabla$ organgs naturgemäß größer ist, also die Hemmung für den Abscheidungsvorgang eine geringere ist.

Solchen abnormen, erst mit steigender Temperatur normal werdenden Verlauf der Stromdichtepotentialkurven beobachtet man auch bei der Abscheidung der Metalle der Eisengruppe aus ihren einfachen Sulfatsalzlösungen.

Hier können wir in Analogie nun die folgende Annahme machen: Die Ionen der Metalle befinden sich in Lösung als komplexe Hydrationen. Bei den allermeisten Metallen erfolgt der Vorgang

$$
\mathrm{Me}\left(\mathrm{H}_{2} \mathrm{O}\right)_{n}{ }^{*} \rightarrow \mathrm{Me} e^{*}+\mathrm{nH}_{2} \mathrm{O}
$$

momentan, bei den Metallen der Eisengruppe bei gewöhnlicher Temperatur so langsam, da $\beta$ man hier ähnlich wie bei der Abscheidung von Kupfar aus komplexen Ionen bei gewöhnlicher Temperatur Verzögerungserscheinungen für den Abschei* dungsvorgang beobachtet. Auch hier beobachtet man mit steigender Temperatur infolge Erhöhung der Reaktionsgeschwindigkeit des langsam verlanfenden Vorgangs Annäherung an das normale Verhalten. Sind nun Metallionen in Lösung, bei denen solch abnormer Verlauf der Strondichtepotentiallikurven vorliegt, so sehen wir leicht, $\mathrm{daB}$ die gemeinsame Abscheidung beider Metalle leichter sein wird, als bei normalem Verlaufe. Bei einer Stromdichte $x x^{\prime}$ (Fig. 2) wird man bei normalem Verlauf der Kathodenpolarisation die $\mathrm{Ab}$ scheidung des zweiten Metalls nicht erreichen, wohl aber bei den oben erwähnten Abweichungen bei einem oder auch bei beiden Metallen.

Alle bisher besprochenen Gesichtspunkte haben nur auf der Annahme gefußt, daß die beiden Metalle in Form eines mechanischen Gemenges zur Abscheidung gelangen.

Bilden jedoch beide Metalle Verbindungen bzw. feste Lösungen, dann wird das edlere Metall die Abscheidung des unedlen erleichtern, oder wie wir sagen, depolarisieren.

Der Grund dieser Erscheinung liegt in der bereits früher erwähnten Tatsache, daß in der Verbindung und auch in einer festen Lösung das unedlere Metall eine geringere Lösungstension hat als in reinem Zustande, sein Kathodenabscheidungspotential dalier nach der edleren Seite verschoben exscheint. Deshalb erfolgt die Abscheidung des unedlen Metalls leichter, d. h. bei niedrigeren Kathodenpotentialen in Form einer Legierung als in reiner form.

So bilden z. B. Kupfer und Zink eime Verbindung $\mathrm{Cu}_{3} \mathrm{Zn}$, die mit Kupfer isomorphe Misch. kristalle liefert, die Gefügebestandteile des Messiugs sind. Weun wir also beispielsweise Messing 
abscheiden wollen, bedienen wir uns, wie die Praxis lehrt, Lösungen, die auf je ein Mol Metallcyanid etwa zwei Mol Cyankalium enthalten. In solchen Lösungen ist das Kupfer um 0,4 Volt edler als das Zink. Die Stromdichtepotentiallsurven verlaufen wie die Kurven 1 und 2 in Fig. 2. Messingabscheidung könnte nach früher Gesagtem also nur aus solchen Iösungen erfolgen bei Stromdichten, die höher als $x x^{\prime}$ sind. Tatsächlich beobachtet man Messingabscheidung bereits von Stromdichten an, bei denen dex Potentialuntersehied noch immer 0,2 Volt beträgt. Wir können also sagen, daß Kupfer die Zinkabscheidung um mindestens 0,2 Volt depolarisiert. In ähnlicher Weise depolarisiert Nickel die Abscheidung des bedeutend unedleren Zinks, so daß aus den einfachen Sulfatlösungen eine Nickel-Zinklegierung abgeschieden wird. Die Abscheidungen werden immer zinkreicher, bis sich schlieBlich nur mehr reines Zink abscheidet. Der Grund dieses Verhaltens ist in der der Uberspannung des Wasserstoffs ganz analogen Erscheinung der Metallüberspannung nu zu suchen, die bei vielen Metallen praktisch Null ist, bei den Metallen der Eisengruppe aber erhebliche Werte annehmen kann, deren kathodische Abschei-

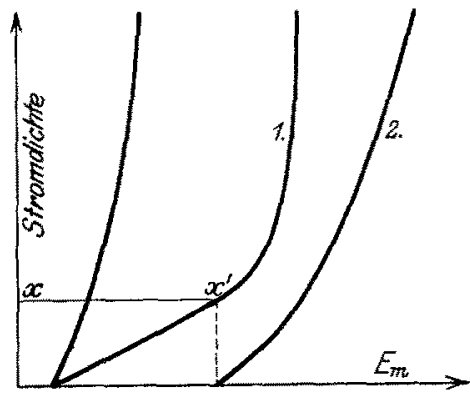

Fig. 2.

dung erschwert und von Stromdichte und Tempew ratur, sowie von der Natur des Kathodenmetalls abhängig ist. Es erleidet also an der zunächst abgeschiedenen Nickel-Zinklegierung die Nickelabscheidung eine erhebliche Überspannung, so dab die Zinkabscheidung der edlere, also leichter vonstatten gehende Vorgang wird.

Eine solche depolarisierende Wirkung, sowie gleichzeitig eine ṫberspannungserscheinung des edlen Metalls sind der Grund, weshalb sich z. B. aus einfachen gemischten Lösungen der Sulfate von Eisen und Kobalt, Kobalt und Nickel und Nickel und Eisen, Legierungen abscheiden, in denen auffallenderweise das unedlere Metall superponiert. Aus den gleichen Gründen kann man die Abscheidung von sonst aus wässerigen Lösungen nicht abscheidbaren Metallen durchführen, wenn man Kathodenmaterialien verwendet, die mit dem abzuscheidenden Metall feste Lösungen und Verbindungen liefern, also auf deren Abscheidung depolarisierend wirken und an denen anderseits Wasserstoff eine erhebliche Utbexspannumg zeigt.

So läbt sich sowohl an Blei- als Zinnkathoden und noch besser an Quecksilberkathoden Natrium abscheiden, das mit den exwähnten Metallen Vexbindungen, bzw. feste Lösungen liefert.

Was run die technische Anwendung der $\mathrm{Ab}$ scheidung von Legierungen anlangt, so wird dicselbe hauptsächlich in der Galvanostegie geübt. Ich erwähnte die Verfahren der Abscheidung von Messing und Tombaküberzügen aus gemischten Cyanidbädern, von Kupfer-Zinnbronzen aus weinsauren Bädern, sowie die Arkasversilberung, wo man statt reinem Silber aus Billigkeitsgründen aus den entsprechenden Cyanidlösungen eine Silber-Cadmiumlegierung niederschlägt. Hier, wo es sich um die Gewinnung ganz dünner, nach $1 /$ !n und $1 / 100$ mm Dicke zählenden Niederschläge handelt, scheint die Abscheidung einheitlicher Produkte leicht durchfuhrbar.

Viel schwieriger ist es, wenn die Aufgabe an uns herantritt, dickere, nach Millimetern und Zentimetern zählende Abscheidungen herzustellen.

Gerade die Bedeutung der Nickelstahle für die Praxis hat den Gedanken nahegelegt, auf elektrolytischem Wege direkt Nickelstahl zu erzeugen. Unter dex Voraussetzung, dab man ein, dem auf thermisch-mechanischem Wege erhaltenen Produkt äquivalentes erhält, läge der Vorteil dieses Verfahrens auf der Hand, weil viel Montagearbeit gespart würde. Leider ist dies nach dem heutigen Stand der Forschung nicht der Fall.

Für die technische Unbrauchbarkeit der aus wässrigen Lösungen erhaltenen Nickel-Eisenlegierungen kommen vornehmlich zwei Momente in Betracht:

Einnal liegt in der Nähe der Abscheidungspotentiale der Nickel-Eisenlegierungen das des Wasserstoffs. Man erhält deshalb ein geringe Mengen Wasserstoff in fest gelöstem Zustand enthaltendes Material; der Wasserstoff härtet wohl das Material, macht es aber zugleich ungemein spröde, fast wie Glas. Zum zweiten steht der Abscheidung in dickeren Platten die Schwierigkeit entgegen, die Konzentrationsverhältnisse im Bade, und damit die $\mathrm{Zu}$ sammensetzung der kathodischen Abscheidung während der ganzen Dauer eines Versuches, der einige Wochen währt), konstant zu erhalten. Man erhält bei aus wässerigen Lösungen abgeschiedenen Nickel-Eisenlegierungen wohl stellenweise ein Gefüge, wie es dem auf thermischem Wege erhaltenen Nickelstahl entspricht; doch beobachtet man auch Stellen, in denen nickelreiche und nickelarme Teile abwechseln. Senkrecht aut die Niederschlagsrichtung kann man deutlich sehen, wie in dicken Platten Schichten verschiedener Zusammensetzung vorliegen. Es ist klar, dal derartige Produkte sich wegen ihrer geringen Festigkeit als technisch whbauchbar erweisen. Erst beim Anlassen solcher Materialien auf Weibglut britt ein Konzentrationsausgleich ein, so dab die Solichtungen rerschwinden. Erst solche Materialien lassen sich techuisch bearbeiten: hiexbei

1) Weil man nur mit niedrigen stromdichten arbei(4) kaun. 
tritt aber starke Biegung der Platten infolge der Wasserstoffabgabe ein.

Trotz dieser ungünstigen Resultate erscheint das Studium der einschlägigen Verhältnisse nicht nutzlos, und eröffnet uns ein weiteres Feld der Forschung. In den Jahren 1894/95 noch wurde in der kritischen Literatur den Versuchen der technischen Darstellung von Elektrolyteisen jede Berechtigung abgesprochen. Und 10 Jahre später erscheint das Elektrolyteisen auf dem Markt, nachdem rastlose Versuche die Schwierigkeiten aus dem Wege geräumt haben. Und so scheinen auch die Versuche, die daliin abzielen, Legierungen auf gleichem Wege im Großen zu erzeugen, vielleieht für die Zukunft nicht nutzlos. Die Bahnen zu skizzieren, die wir hierbei zu gehen haben, ist der Zweck dieser Zeilen.

\section{Besprechungen.}

Pagels, J. L., Eintuhrung in die Gesehichte der Medizin in 25 akademischen Vorlesungen. Zweite Aufl., durchgesehen, teilweise umgearbeitet und auf den heutigen Stand gebracht von Karl Sudhoff. Berlin, S. Karger, 1915. XVI, 616 S. Preis geh. MT. 20,-, geb. M. $22,-$.

Unter dem bescheidenen Titel einer zweiten Auflage erscheint hier tatsachlich eine völiffe Neubeabcilung der Pagelsehen Einfuhrung durch den Altmeister der Geschichte der Medizin Sudhoff. So wie das Buch jetzt vor uns liegt, kann es für die meisten Arzte vollständig eine Geschichte ihrer Wissenschaft ersetzen; für den Nichtmediziner, sei er Philolog, Historiker oder Kulturforscher, muB es ebenfalls als eine Quelle gut verständlicher Belehrung bezeichnet werden. Mit Recht hatte der Verleger darauf gedrungen, dab der Charakter fortlaufender Vorlesungen gewahrt bleibe. Gerade dadurch wirkt diese Lektüre durchweg anregend, statt zu ermüden. Von Seite zu Seite und Bogen zu Bogen wird man immer mehr gewahr, dab der jetzige Verfasser mit dem Rüstzeug eines gut gebildeten Historikers arbeitet und dabei doch durchweg ein ärztliches Ver ständuis an den Tag legt, wie es nur durch jahrzehntelanges, nachdenkendes Arbeiten auf dem Gebiete der praktischen Medizin exworben werden kann.

Die beiden ersten Vorlesungen über das vorklassische Altertum sind vollig neu. Sie hatten vor zehn Jahren in dieser Weise uberhaupt noch nicht geschrieben werden können. Sie behandeln die Knochenfunde und Zeichnungen der Steinzeit von Nordwestund Zentraleuropa, ferner die alte Medizin von China und von Japan. Von Japan wird ansdrieklicli botont, daB seit 1616 enropüischer FinfluB vorhanden ist, und seit 1867 mit deutschem Kalbe gepfligt wird. Bei den Aateken war wie bei den Agyptern die Medizin Geheimwissenschaft der Priester. Sehr bemerkenswert fiur die Erfassung eventueller Kulturzusammenhänge nach Asien hin ist die astrologisohe bzw. die $K a$ lenderprognostik und diagnostik. Wie wir im Hellenismus auf chaldäiselere, d. h. babyloniseher Tradition Zusammenhänge zwisehen Körperteilen uud Himmelszeichen finden, so auch bei den Azteken. Auch die Arzneiverordnung stand unter Himmelsgewalten, wie am Euphrat und am Nil. Natïlich spielte auch die $\mathbb{S} y m$. pathie wie bei allen Naturvölkern eine Rolle. Die
Zahl der Arzneimittel war recht groß. Man verstand Wunden mit Geschick zu behandeln und den Aderlap mittels Obsidinnessev auszufüren; selbst die Wundnaht (mittels Haaren), die Kenninis von erhartenden Verbänden und die Reposition von Laxationen sind nachweisbax. Auf dem Gebiete der Frauenheilkunde tinden wir die Behandlung falsoher Kindslagen, Embryotomie, Wöchnerinnenpflege, Kindspflege, hygienische Kindererziehung (Bäder, Mundpflege, Nahrungsmittelkontrolle, hochentwickelte Gymnastik).

Bei der Besprechung der altindisohen Medizin gelt Sudhoff you der Tatsache aus, daB die beiden Fruptarrte Atreya und Suschruta im sechsten vorchristlichen Jillrhundert gelebt haben, also ein Jahrhundert vor dem Zeitalter des Hippolrates. Mithin können sie nichts aus der griechischen Medizin entlehnt haben, wie zeitweise behauptet worden ist. Der wichtigste Fund fur die Erforschung der altindischen Medizin ist das Bower-Manuskript, welches der in England als Sprachforscher lebende Schwabe $R$. Hoernle in den Jahren 1893-1912 bearbeitet, übersetzt und erkiärt hat. Es ist auf Birkenbast in den Jahren 350-375 nach Christo geschrieben. Natürlich wirtschaftet die altindigehe Medizin mit Zaubergesängen, aber sie entLatlt doch recht Bemerkenswertes, so viele Hunderte von Arzneimitteln, pflanzlichen, tierischen und mineralisehen Ursprung's, toxikologische Einzelheiten, Chirurgisches (Aderlaß, Sehröpfkopf, Blutegel, Wundbehandlung, Tumorexstirpation, Laporotomie, Steinschnitt, Rininoplostik), hochentwickelte Gesundheitspflege.

Unsere Kenntnis der Medizin bei den alten Babyloniern beruht lauptsäehlich auf den aufgefundenen Resten der Bibliothek des Assyrerkönigs Sardanapal (Assurbanipal), die zu Ende des VII. Jahrhunderts sich in Ninive befand. In London befinden sich ungefalis 900 und in Berlin 1000 medizinische Keilschriftexte dieser Bibliothek. Allerdings ist die Hauptmenge davon noch unübersetzt. Soviel steht fest, daß wir für die nächsten Jahre eine Fülle von Angaben über die gewöhnlichsten damaligen Krankheiten und deren Behandlung ibersetzt bekommen werden. Neben Zaubersprüchen spielten dabei arzneiliche und chirurgische Mabnahmen eine Rolle. Dem Arste, der einen chirurgischen Kunstfehler befangen hat, wird das Hädeabhauen angedroht.

Der Datierbarkeit nach rejcht die medizinische Literatur der Ägypter weit über die der Babylonier hinaus, sicher bis ins dritte Jahrtausend vor unserer Zeitrechnung zurück. Der bekannte Papyrus Ebers ist keineswegs das älteste medizinische Manuskript jener Epoche, aber doch das wichtigste. Fr enthält die Behandlung der Leiden der verschiedenen Körperteile. Zwei Berliner Papyri und ein Londoner ergänzen ihn wesentlich. Sicher ist, dab bei der Krankenuntersuchung, Inspektion, Patpation, ja selbst eine Art Auskultation eine Rolle spielten; fermer wurden wle Ansscheidungen beachtet, besonders Urin, Solweip, Ructus und Fatus. Brech- und Abfuhrmittel spielten eine große Rolle.

Die dritte und vierte Vorlesung wenden sich den altell Griechen zu und besprechen mit Wärme und eingehendstem Sachverständnis die klassisehe Periode der alten Medizin. Mit der Begriundung der akademischen Schule ist die grundsätzliche Einfihrung methodisother Unterswchungen an mensohlichen Leichen verknupft. Damit erweiterte sieh naturgemẩ der Wirkungskreis der operativen Chirurgie. In Alexandria wurden einzelne Verbrecher einem Bericht von Celsus zufolge nicht nach dem Tode, sondern bei Leb- 